EPJ Web of Conferences 41, 12014 (2013)

DOI: $10.1051 /$ epjconf/20134112014

C Owned by the authors, published by EDP Sciences, 2013

\title{
Coherent Lensless imaging with Ultra-Broadband Light Sources
}

\author{
S. Witte ${ }^{1}$, V. T. Tenner ${ }^{1}$, D. W. E. Noom ${ }^{1}$ and K. S. E. Eikema ${ }^{1}$ \\ ${ }^{1}$ LaserLaB, VU University Amsterdam, De Boelelaan 1081, 1081 HV Amsterdam, The Netherlands
}

\begin{abstract}
We demonstrate a method for efficient lensless imaging using ultrabroadband light sources. By using a pair of time-delayed, coherent pulses in a Fouriertransform scheme, spectrally resolved diffraction patterns are obtained throughout the entire spectrum of the incident light source. We perform a proof-of-principle experiment using an octave-spanning visible light source, and obtain images of a holographic test sample with near-diffraction limited resolution. Our approach provides a promising route towards efficient high-resolution imaging using table-top high-harmonic soft-Xray sources.
\end{abstract}

\section{Introduction: High-resolution coherent lensless imaging}

High-resolution imaging is an essential tool for studying nature at the smallest possible scale. In fields ranging from cell biology to condensed matter physics, a large amount of knowledge can be gained through visualization of the smallest possible structures in a system. Due to the inherent resolution limitations of visible light, the use of XUV and X-ray sources for high-resolution imaging applications is rapidly gaining popularity. Various types of novel X-ray sources are being developed around the world, ranging from $\mathrm{km}$-size X-ray free electron lasers (XFELs) to compact table-top high-harmonic sources. As the development of good-quality X-ray imaging systems is challenging, the use of lensless imaging methods [1-3] is an interesting alternative for the practical realization of high-resolution X-ray microscopes.

In principle, table-top high-harmonic generation (HHG) sources seem ideally suited for the development of soft-X-ray microscopes [4], as they are compact in size, highly coherent, and costeffective compared to XFELs. However, the intrinsically ultra-broadband spectrum in which HHG is generated forms a significant limitation, since lensless imaging typically requires a quasimonochromatic source. While state-of-the-art HHG sources achieve significant flux [5], the need to spectrally filter out a narrow bandwidth leads to an orders of magnitude lower flux that can effectively be used for imaging applications. Improved algorithms can alleviate the requirements [6], but only work for a limited fractional bandwidth.

We find that, by introducing methods from Fourier-transform spectroscopy in lensless imaging, we can perform high-resolution imaging with ultra-broadband coherent light sources. This approach is general and can be applied to any type of coherent lensless imaging such as coherent diffractive imaging, Fresnel-domain diffractive imaging, and Fourier-transform holography. 

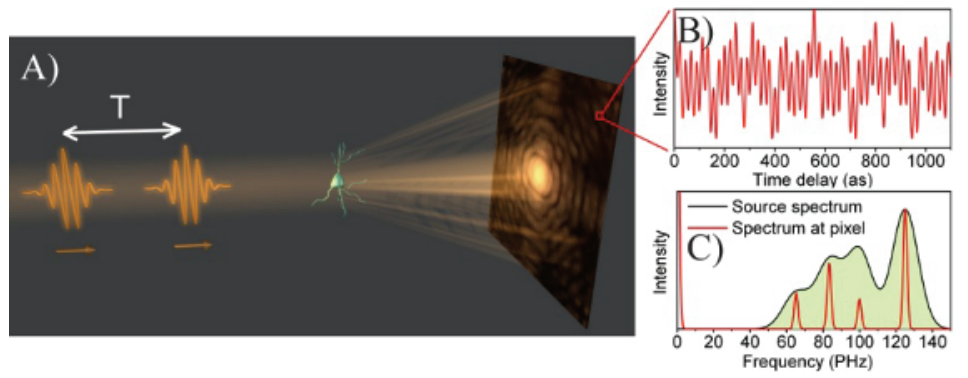

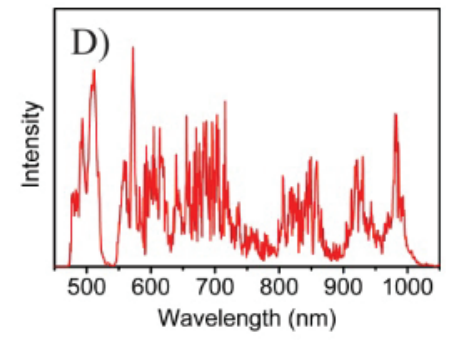

Fig. 1. A) Concept of two-pulse Fourier-transform lensless imaging. A lensless imaging experiment is performed with two coherent pulses, while scanning the time delay between them. B) Simulation of the signal on a single pixel as a function of the time delay. C) A Fourier transformation of the signal in B directly resolves the spectral components scattered onto that specific pixel. D) Spectrum of the octave-spanning white-light continuum used in our imaging experiments.

\section{Two-pulse Fourier-transform lensless imaging}

The concept is shown in Fig. 1A. A pulse is split into two time-delayed, coherent replicas using an interferometer, and this pulse pair is then used in a standard lensless imaging experiment. By capturing images while scanning the delay between the pulses, a Fourier-transform spectrum of the diffracted light is recorded on each pixel of the detector, directly revealing which spectral components are scattered onto that specific pixel (Fig. 1C).

By Fourier transforming the time delay scan for each pixel (Fig. 1B), a spectrally resolved stack of images is obtained, containing quasi-monochromatic diffraction patterns for all spectral components in the pulse bandwidth. The achievable spectral resolution is only limited by the scan length: no limitations exist for the source spectral bandwidth, which does not even have to be known in advance.

We experimentally implement two-pulse Fourier-transform imaging using an octave-spanning white-light continuum (WLC) as the light source (Fig. 1D). The WLC is generated in a photonic crystal fiber, pumped by $\sim \mathrm{nJ}$ pulses from a $10 \mathrm{fs}$ Ti:Sapphire oscillator. To generate the two coherent, time-delayed replica pulses, these WLC pulses are sent into a Michelson interferometer, of which one mirror is mounted on a closed-loop piezo-stage to introduce a delay. This pulse pair is then used to illuminate a sample, and the diffracted light is recorded with a 14-bit CCD camera. For one experiment, we record 500 individual images while increasing the time delay in $0.67 \mathrm{fs}(200 \mathrm{~nm})$ steps. After image acquisition, we Fourier-transform the time delay scan for each pixel, and reconstruct images for each component in Fourier space.

\section{Results}

As a proof-of-principle experiment, we perform two-pulse Fourier-transform imaging on a test sample suitable for lensless holography [2] (Fig. 2A), so that a spatial 2D-Fourier transform suffices for a first image reconstruction. Lensless imaging with an ultra-broadband spectrum results in a strongly radially smeared diffraction pattern (Fig. 2B), as the scattering angle is directly proportional to the wavelength of the light. As a result, interference fringes are washed out, and sharp diffraction patterns become blurred to the point where image reconstruction becomes impossible. Figure $2 \mathrm{C}$ shows a spectrally resolved diffraction pattern of the same structure, obtained using our two-pulse Fourier-transform approach. Sharp diffraction features are resolved throughout the entire image, and the pattern is equivalent to a diffraction pattern recorded with a monochromatic source.

The reconstructed images resulting from the broadband and spectrally-resolved diffraction patterns are shown in Figs. 2D and 2E, respectively. The broadband image shows strongly distorted structures which are smeared out across a large part of image space, in which the original structure is barely recognizable. In contrast, the image resulting from the spectrally resolved diffraction pattern 
is a sharp, well-resolved reconstruction of our ' $\mathrm{nr}$. 4' test sample. Figure $2 \mathrm{~F}$ shows an overlay for three different spectral components extracted from the two-pulse Fourier-transform image data. This specific sample acts as a simple scatterer for all wavelengths, so that the different wavelength images only differ in the size of their field-of-view, and can be averaged to further improve image contrast. For wavelength-dependent samples, this approach forms a direct way of robust spectroscopic imaging, as diffraction patterns are obtained for all wavelengths simultaneously. This provides a natural way of obtaining spectrally-resolved images at e.g. different absorption edges. Another advantage of the two-pulse Fourier-transform approach is its robustness against spectral intensity fluctuations and stray light hitting the detector, as the signal is encoded as a modulation in the time delay scan. It should be noted that such spectral fluctuations were prominently present in our WLC spectrum.

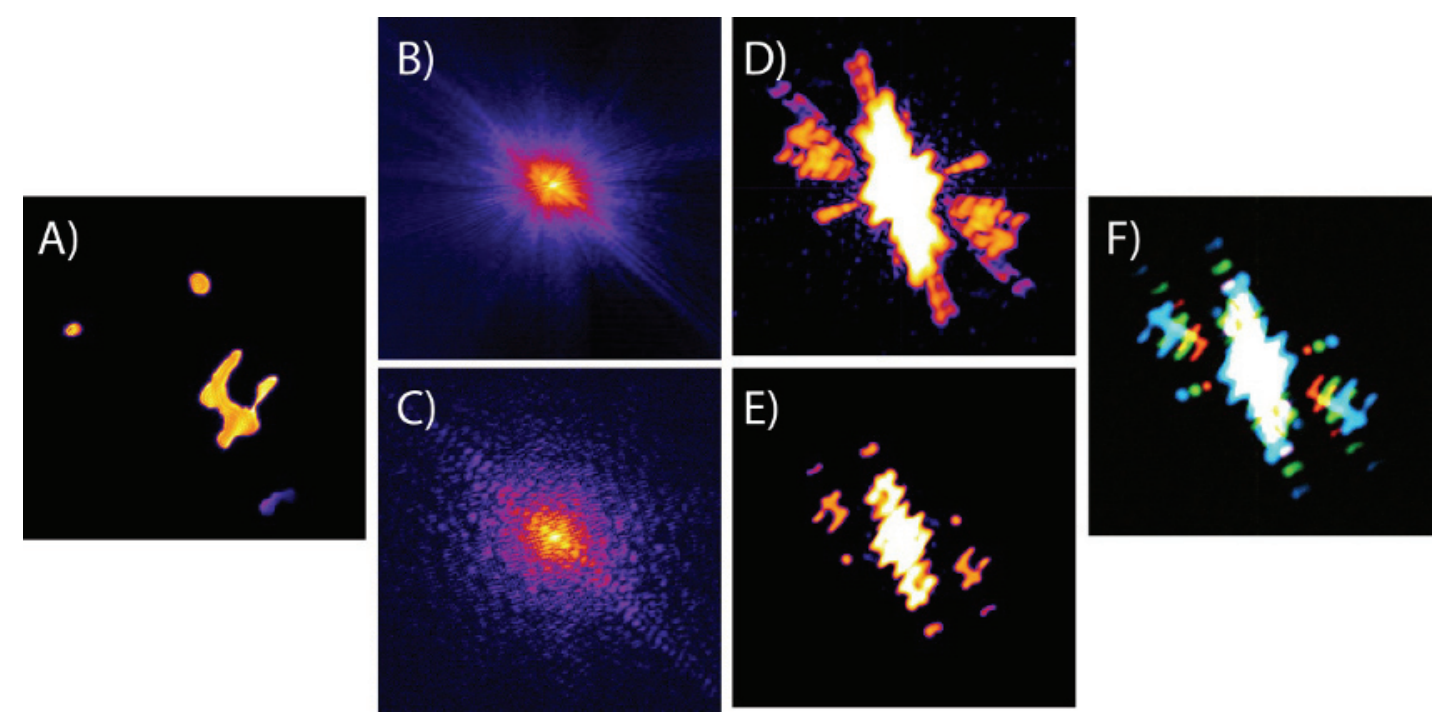

Fig. 2. A) Wide-field microscope image of the transmission test sample used in the experiments. B) Coherent diffraction pattern of the sample, recorded with an octave-spanning spectrum. C) Diffraction pattern of the same object, obtained with two-pulse Fourier-transform imaging. A spectral bandwidth of $\Delta \lambda / \lambda=1 / 125$ was filtered numerically. D) 2D-Fourier transform of B, showing strongly distorted features. E) 2D-Fourier transform of C, in which the test structure is now clearly resolved. F) Overlay of the reconstructed images from three selected spectral components in the two-pulse Fourier-transform scan.

Our results show the feasibility and promise of two-pulse Fourier-transform lensless imaging. It enables highly efficient imaging, making optimal use of the full spectral bandwidth of any coherent light source. The practical implementation at short wavelengths relies on the generation of two timedelayed pulses, which has already been demonstrated at XUV wavelengths [7]. Therefore, we believe that two-pulse Fourier-transform lensless imaging forms a promising framework for highresolution soft-X-ray microscopy with table-top HHG sources.

[1] J. Miao et al., Nature 400, 342, (1999).

[2] I. McNulty et al., Science 256, 1009 (1992).

[3] H.N. Chapman, K.A. Nugent, Nat. Photonics 4, 833 (2010).

[4] R.L. Sandberg et al., Opt. Lett. 34, 1618 (2009).

[5] T. Popmintchev et al., Proc. Natl. Acad. Sci. USA 106, 10516 (2009).

[6] B. Abbey et al., Nat. Photonics 5, 420 (2011).

[7] N. de Oliveira et al., Nat. Photonics 5, 149 (2011). 